\title{
CARACTERÍSTICAS FÍSICO-QUÍMICAS DEL CHARQUI DE LLAMA
}

\author{
Physicochemical Characteristics of Llama Jerky \\ Willy Mamani-Linares ${ }^{1,2}$, Faustina Cayo R. ${ }^{1}$
}

\section{RESUMEN}

\begin{abstract}
El objetivo de este trabajo fue estudiar las características fisicoquímicas del charqui de llama. Se muestrearon 20 piezas de charqui de llama, de unos 450 a 500 g cada uno, comprados de diferentes mercados y carnicerías de la ciudad de Oruro, Bolivia. Se determinó la humedad, grasa, proteína, ceniza, colesterol, minerales, perfil de ácidos grasos, colágenos totales y solubles, sustancias reactivas al ácido tiobarbitúrico, color, $\mathrm{pH}$, actividad de agua, capacidad de retención de agua y fuerza de corte. El charqui de llama se caracteriza por contener altos niveles de proteína, baja humedad y actividad de agua, mientras que el contenido de minerales, la relación ácidos grasos poliinsaturados/saturados y el contenido de ácido linoleico conjugado es comparable a los encontrados para charqui de alpaca y bovino. El charqui mostró una buena relación de n-6/n-3 (3.70) y altos niveles de ácidos grasos deseables $(70.27 \%)$. Los valores de los parámetros tecnológicos del charqui de llama están en el rango reportado para los charquis convencionales.
\end{abstract}

Palabras clave: características fisicoquímicas, charqui, llama

\section{Abstract}

The aim of this study was to determine the physicochemical characteristics of llama jerky. Twenty pieces of jerky, each 450 to $500 \mathrm{~g}$ were purchased from various markets and local butchers in the Oruro city, Bolivia. It was determined moisture, fat, protein, ash, cholesterol, minerals, fatty acid profile, total and soluble collagen, Thiobarbituric Acid Reactive Substances, colour, $\mathrm{pH}$, water activity, water holding capacity and WarnerBratzler shear-force. Llama jerky was characterized by a high protein content, low moisture and water activity, while mineral composition, polyunsaturated to saturated fatty acids ratio and conjugated linoleic acid content were comparable to those found for alpaca and beef jerky. Llama jerky showed a good n-6/n-3 (3.7) ratio and high levels of desirable fatty acids $(70.27 \%)$. Technological quality parameters of llama jerky were in the range reported for more conventional jerky type.

Key words: physicochemical characteristics, jerky, llama

\footnotetext{
${ }^{I}$ Escuela de Graduados, Facultad Ciencias Veterinarias, Universidad Austral de Chile, Valdivia, Chile.

2 Email: willymlmvzupea_2@hotmail.com
} 
El charqui es uno de los productos más antiguos de la carne que se conservan por salazón y secado. Es relativamente fácil de procesar, tiene un sabor típico, y no necesita refrigeración durante su distribución comercial debido a su baja actividad de agua (aw). El charqui, además de ser un producto nutritivo (rico en proteínas y bajo en grasa), es estable a medio ambiente y tiene una gran demanda en el mercado.

Tradicionalmente, el charqui se hace de carne cortada en rodajas finas que son marinadas y secadas. La estabilidad se logra al secar el charqui hasta alcanzar $0.70-0.85$ de actividad de agua (aw) (Quinton et al., 1997). El charqui es magro, y puede ser también seco y quebradizo, fácil de masticar, pero tienen un color no deseado cuando es sobresecado (Leistner, 1987).

Algunos tipos de charqui que presentan textura suave pero con alta actividad de agua (aw) y alto contenido graso, tienden a presentar mayor actividad microbiana y oxidación de lípidos. Esta última es una de las mayores causas de deterioro de la calidad en el charqui, y es más rápido en carne cocida que en carne cruda (Tichivangana y Morrissey, 1985). Además, una serie de factores extrínsecos o ambientales como la temperatura, la luz y el nivel de oxígeno, influyen la oxidación de los lípidos en los alimentos cárnicos.

Se puede preparar una gran variedad de charquis empleando carne de vacuno, cerdos, aves y otras especies (Lonnecker et al., 2010). El charqui hecho a base de res ha sido el de mayor uso; sin embargo, charqui hecho a partir de carne de llama, equino, cerdo, y otras carnes son muy populares. El charqui de llama en la zona del Altiplano es procesado de una forma artesanal y por lo tanto, la calidad se ve afectada durante el almacenamiento. Es así que el objetivo de este trabajo fue estudiar las características fisicoquímicas de charqui de llamas.

\section{Muestras}

Muestras de charqui de llama $(\mathrm{n}=20)$ de 450-500 $\mathrm{g}$ fueron adquiridas en varios mercados y carnicerías de Oruro, Bolivia. Todos los charquis procedían de llamas faenadas en el matadero de Turco y fueron elaborados y procesados por microempresarios instalados en la localidad. Un máximo de dos muestras fueron compradas de la misma marca y de la misma fecha de envasado. No se intentó identificar el origen anatómico o la edad del animal del cual procedieron las muestras. Todas las muestras fueron almacenadas a temperatura del medio ambiente en los días previos del análisis, manteniendo las condiciones de almacenamiento de los lugares de expendio. $\mathrm{El}$ análisis de laboratorio (con excepción de TBARS) de la muestras se realizó en el transcurso de los 30 días posteriores a la compra de las muestras.

\section{Análisis de Composición Química}

Composición proximal. Las muestras de charqui fueron molidas y homogenizadas previamente al análisis. Las muestras se analizaron en duplicado. La humedad se determinó con el método oficial AOAC 950.46, de secado al horno (AOAC, 1996), la proteína bruta con el método oficial AOAC 981.10, método Microkjeldahl Gerhardt que determina $\mathrm{N}$ en carne (AOAC, 1996), la grasa con el método de la norma Chilena NCh 1370/3/ 1970, y las cenizas con el método oficial AOAC 920.153, método directo de calcinación en mufla (AOAC, 1996).

Análisis de colesterol. Los lípidos de la muestra (10 g) se extrajeron con cloroformometanol (2:1), según metodología descrita por Folch et al. (1957). Una alícuota del extracto lipídico ( $3 \mathrm{ml})$ fue secado en nitrógeno y saponificado con $12 \% \mathrm{KOH}$ etanólico (10 ml) durante 15 min a $80{ }^{\circ} \mathrm{C}$ de acuerdo a Bohac et al. (1988). Después de enfriar y añadir 5 $\mathrm{ml}$ de agua destilada, el material 
insaponificable se extrajo dos veces con 10 $\mathrm{ml}$ de hexano. Tres mililitros del extracto fue secado en nitrógeno, redisuelto en $3 \mathrm{ml}$ de la fase móvil, y el colesterol fue cuantificado por HPLC/UV.

Análisis de ácidos grasos. Los lípidos de la muestra fueron extraídos de acuerdo a Folch et al. (1957). El extracto de lípidos fue convertido a ésteres metílicos de ácidos grasos (FAME) usando la metodología descrita por Bohac et al. (1988). La separación y cuantificación se hizo utilizando un cromatógrafo de gases (Shimadzu GC-148 con detector FID) equipado con una columna capilar HR-SS-10 $(100 \mathrm{~m}, 0.35 \mathrm{~mm}$ de diámetro interior y $0.32 \mu \mathrm{m}$ de espesor de película, Shinwa Inc). Los ácidos grasos individuales fueron identificados por comparación de sus tiempos de retención con los estándares de la mezcla de ácidos grasos Sulpeco 37 (Sigma Chemical Co. Ltd, Poole, UK). El éster metílico del ácido nonadecanoico (19:00 ME) se utilizó como estándar interno. Los ácidos grasos se expresaron como porcentaje del total de ácidos grasos identificados y agrupados como ácidos grasos saturados (SFA), monoinsaturados (MUFA) y poliinsaturados (PUFA). Ácidos grasos menores y no resueltos no fueron reportados.

Contenido de colágeno. Las muestras molidas $(5 \mathrm{~g})$ se calentaron por $70 \mathrm{~min}$ a $77^{\circ} \mathrm{C}$ en solución de Ringer 1/4-strength y dividido en fracciones de residuos sobrenadantes, siguiendo el procedimiento de Hill (1966). Cada fracción se hidroliza de forma individual en $6 \mathrm{~N} \mathrm{HCl}$ durante 6 horas a $1 \mathrm{~atm}$ de presión y $102{ }^{\circ} \mathrm{C}$. Los niveles de hidroxiprolina se determinaron según Bergman y Loxley (1963). El contenido de colágeno (mg/g) se calculó a partir de los niveles de hidroxiprolina por los valores de conversión de 7.25 y 7.52 para el colágeno soluble e insoluble, respectivamente (Cross et al., 1973).

Contenido de minerales. Alícuotas de 0.25 $\pm 0.01 \mathrm{~g}$ de la muestra de carne fueron pesadas y digeridas con $\mathrm{HNO}_{3}$ concentrado en recipientes de vidrio con tapón de rosca bien cerrado. El contenido de minerales se determinó por espectrofotometría de absorción atómica, método 975.03 (AOAC, 1996).

\section{Características Tecnológicas}

Actividad de agua (o actividad acuosa). Tres piezas de cada muestra de charqui se seleccionaron y se cortaron en trozos pequeños y se homogenizaron. La actividad se determinó con un medidor de actividad de agua (AQS-2, Nagy mess system, Alemania), calibrado a temperatura ambiente $\left(20^{\circ} \mathrm{C}\right)$ con agua destilada (aw $=0.999)$ y solución saturada de $\mathrm{NaCl}(\mathrm{aw}=0.756)$ y $\mathrm{KCl}(\mathrm{aw}=$ $0.853)$.

Color de la carne. Se determinó el color de la superficie del charqui en trozos por medio del espectrocolorímetro HUNTERLAB con escala Lab y con una longitud de onda entre 400 y $700 \mathrm{~nm}$, con las mediciones estandarizadas con respecto a una placa de calibración en blanco $\left(L^{*}=89.2, a^{*}=0.921\right.$, $\left.b^{*}=0.783\right)$. La medición del color se hizo por triplicado. Se determinó el valor " $L$ *" (luminosidad), "a*" (tenores de rojo-verde) y "b*" (tenores de amarillo-azul).

$p H$. Se midió con un peachímetro con electrodo de pincho, marca HANNA, modelo HI 9025. Se empleó $3 \mathrm{~g}$ de charqui molido, al cual se agregó $27 \mathrm{ml}$ de agua destilada. Se mezclaron con un homogeneizador (T25 basic, IKA, Malasia) y se registró el pH por duplicado. El medidor de $\mathrm{pH}$ se calibró al inicio y cada diez mediciones con buffer estándar de pH 4.0 y 7.0 a $25^{\circ} \mathrm{C}$.

Sustancias reactivas al ácido tiobarbitúrico (TBARS). Se midió en forma mensual durante seis meses de almacenamiento, de acuerdo al método descrito por Sinnhuber y Yu (1977). La absorbancia se midió con un espectrocolorímetro (DU-640, Beckman Instruments, Inc., Fullerton, CA) en una longitud de onda de $531 \mathrm{~nm}$. Los valores de TBARS fueron expresados como miligramos de malonaldehido producidos por 
kilogramo de muestra. El índice de ditiobarbitúrico se determinó por triplicado en cada muestra.

Capacidad de retención de agua. Se pesó $15 \mathrm{~g}$ de charqui cortados en trozos y se añadió agua en una proporción charqui: agua de 1:10. Se dejó en reposo por $18 \mathrm{~h}$ a temperatura ambiente, el agua se escurrió con un colador fino y se pesó el charqui rehidratado, determinándose el porcentaje de ganancia de peso. Porciones de $15 \mathrm{~g}$ de charqui rehidratado se pesaron de forma individual dentro de bolsas de plástico de cierre hermético en un baño de agua a $75^{\circ} \mathrm{C}$ durante $30 \mathrm{~min}$, hasta que la temperatura interna alcanzó $70^{\circ} \mathrm{C}$, y seguidamente se enfriaron hasta los $4{ }^{\circ} \mathrm{C}$. Las muestras fueron secadas con papel toalla y pesadas. La pérdida de cocción se expresó como el porcentaje de pérdidas relacionadas con el peso inicial (Honikel, 1998).

Textura. La fuerza de corte $\left(\mathrm{kg} / \mathrm{cm}^{3}\right)$ se midió con un equipo Warner-Bratzler, marca Salter. Para esto, un trozo de cada muestra es sometido a cocción como se describe en el punto anterior, seccionado transversalmente (en dirección paralela a las fibras musculares) en secciones de $0.5 \times 2.0 \times 4.0 \mathrm{~cm}$ para medir textura por quintuplicado para cada muestra.

\section{Análisis Estadístico}

Se empleó estadística descriptiva, expresada como promedios y desviaciones estándares.

\section{Resultados y Discusión}

El Cuadro 1 muestra el análisis proximal de charqui de llama. El 19.05\% de humedad fue ligeramente superior a los valores de 12.9 a $17.3 \%$ en charqui de alpaca descritos por Salvá (2009). Los resultados indican que el charqui de llama tiene valores inferiores de humedad que el de bovino, que va entre 24.2 a 34.9\% (Carr et al., 1997; Konieczny et al., 2007; Yang et al., 2009). El contenido protei- co fue superior al $49.0 \%$ reportados en charqui de alpaca (Salvá, 2009), pero similar al $63.6 \%$ en charqui de bovino (Konieczny et $a l .$, 2007). El contenido de grasa fue, asimismo, mayor que en el charqui de alpacas (Salvá, 2009), pero menor al charqui de bovino $(9.3 \%$, Carr et al., 1997) y de cerdo (17.8\%, Choi at al., 2008). Por último, el contenido de ceniza fue inferior al encontrado en alpacas (30.0\%, Salvá et al., 2009), pero superior al contenido en charqui de bovino (4.1\%), cerdo (5.1\%), emú (5.5\%) y pavo (5.3\%) (Carr et al., 1997; Choi et al., 2008).

El promedio de colesterol en charqui de llama fue de $139.6 \mathrm{mg} / 100 \mathrm{~g}$, no habiéndose encontrado valores referenciales para carne deshidratada de camélidos y otros rumiantes; sin embargo, se reportan niveles de 51 y $56 \mathrm{mg} / 100 \mathrm{~g}$ de colesterol en carne fresca (músculo L. dorsi) de alpacas y llamas, respectivamente (Cristofanelli et al., 2004), y de 52 y $93 \mathrm{mg} / 100 \mathrm{~g}$ en $L$. dorsi y grasa renal de llamas (Coates y Ayerza, 2004). El contenido de colesterol es más alto en carne de llama que de alpaca; sin embargo, estos valores son semejantes al contenido de colesterol en carne de camellos $(42-47 \mathrm{mg} / 100 \mathrm{~g}) \mathrm{y}$ bovinos (56.3 - $57.3 \mathrm{mg} / 100 \mathrm{~g}$ ) (RIRDC, 2007; Duckett et al., 2009), pero inferiores a la del ovino (65.9-67.9 mg/100 g) (Costa et al., 2009).

El contenido de colágeno fue de 5.33 $\mathrm{mg} / \mathrm{g}$, siendo soluble el $15.3 \%$. Este valor puede ser comparado con el estudio de Salvá (2009) en charqui de carne de alpaca donde se encontró valores de 3.07 y $3.83 \mathrm{mg} / \mathrm{g}$ para charquis procedentes de diferentes zonas, $\mathrm{y}$ de $4.92 \mathrm{mg} / \mathrm{g}$ para carne fresca.

En el Cuadro 2 se presenta el perfil de ácidos grasos del charqui de llama. El principal ácido graso en la grasa intramuscular fue C18:1n-9 (30.39\% del total de ácidos grasos), seguido por C16:0 (23.38\%) y C18:0 (20.00\%). El Cuadro 3 muestra la proporción de los ácidos grasos (\%) en la grasa intramuscular. Los ácidos grasos saturados (SFA) representan el $49.25 \%$ del total de 
Cuadro 1. Composición de charqui de llama, expresado en porcentaje sobre base húmeda

\begin{tabular}{lccc}
\hline Parámetro & Promedio & D.E. & Rango \\
\hline Humedad $(\%)$ & 19.05 & 2.57 & $16.7-22.9$ \\
Grasa $(\%)$ & 6.30 & 0.75 & $5.5-7.4$ \\
Proteína $(\%)$ & 66.12 & 2.11 & $64.6-69.8$ \\
Ceniza $(\%)$ & 8.54 & 0.67 & $7.8-9.5$ \\
Colesterol (mg/100g) & 139.58 & 5.05 & $131.7-145.6$ \\
Colágenos totales $(\mathrm{mg} / \mathrm{g})$ & 5.33 & 0.35 & $5.1-5.9$ \\
Colágeno soluble $(\mathrm{mg} / \mathrm{g})$ & 0.82 & 0.25 & $0.6-1.2$ \\
Colágeno soluble $(\%)$ & 15.30 & 1.80 & $12.4-16.9$ \\
\hline
\end{tabular}

ácidos grasos, los ácidos grasos monoinsaturados (MUFA) el $41.37 \%$ y los poliinsaturados (PUFA) el $8.90 \%$. La grasa intramuscular tiene una relación de 0.18:1 para PUFA:SFA, valor intermedio para valores de carne de rumiantes, mientras que la relación de n-6: n-3 en grasa intramuscular de alpaca es 3.70:1, que es mayor en comparación con las proporciones de 2 y 3 reportadas para carne de bovinos y ovinos (USDA, 2008). La relación PUFA:SFA y n-6:n-3 en grasa intramuscular está próxima a las recomendadas por BDH (1994) que es 0.4 y 4 , respectivamente.

El perfil de ácidos grasos en la carne de alpaca ha sido reportado (Salvá, 2009). En el charqui se encuentran cerca de 20 ácidos grasos, siendo los principales C18:1 n-9 (30.58\% del total de ácidos grasos), C16:0 (24.02\%) y C18:0 (22.98\%), donde los SFA, MUFA y PUFA representaron el 55.5, 35.5 y $8.8 \%$ del total de ácidos grasos, en tanto que en carne fresca se reportan más de 30 ácidos grasos, siendo los principales C18:1 n-9 (24.24\%), C16:0 (22.01\%) y C18:0 (19.82\%), donde los SFA, MUFA y PUFA representaron el 51.2, 37.1 y $11.7 \%$. La grasa intramuscular de alpaca tiene una relación de 0.26:1 para PUFA:SFA, y la relación de n-6: n-3 en grasa intramuscular es de 3.74:1. Ade- más, el contenido de ácido linolénico conjugado (CLA) fue $1.2 \%$ de los ácidos grasos totales.

El contenido de minerales se presenta en el Cuadro 4. El NA y K son los de mayor nivel mientras que el manganeso y cobre son los que se presentan en menor proporción. Salvá (2009) reporta niveles elevados de sodio en charqui de alpaca (11256 a $16708 \mathrm{mg} / 100$ $\mathrm{g}$ ), niveles moderados de fósforo y magnesio (467 y $215 \mathrm{mg} / 100 \mathrm{~g}$, respectivamente) y niveles bajos de cobre y manganeso $(0.025 \mathrm{y}$ $0.2 \mathrm{mg} / 100 \mathrm{~g}$, respectivamente). Sin embargo, se reporta que la carne fresca de llama y alpaca tienen altos niveles de potasio, lo cual también es registrado en camellos (Kadim et al., 2006). El contenido de potasio y calcio en carne de llamas es mayor que en la carne de alpaca. Por otro lado, la carne del bovino presenta un alto contenido de potasio, magnesio y calcio y bajos niveles de zinc (E1Faer et al., 1991; Kadim et al., 2006, Göncü Karakök et al., 2010). También se reporta que el contenido de hierro en carne de llamas es mayor que en la carne bovina (Duckett $e t$ al., 2009).

Los resultados de los parámetros de calidad tecnológica del charqui de llama se muestran en el Cuadro 5. El pH fue de 5.96, bastante similar a los valores de $\mathrm{pH}$ de char- 
Cuadro 2. Contenido de ácidos grasos de charqui de llama, expresado como porcentaje del total de ácidos grasos

\begin{tabular}{|c|c|c|}
\hline Ácidos grasos & Promedio & D.E. \\
\hline C6 & 0.03 & 0.02 \\
\hline $\mathrm{C} 8$ & 0.07 & 0.01 \\
\hline C9 & 0.01 & 0.01 \\
\hline $\mathrm{C} 10$ & 0.21 & 0.03 \\
\hline $\mathrm{C} 11$ & 0.01 & 0.01 \\
\hline $\mathrm{C} 12$ & 0.26 & 0.05 \\
\hline $\mathrm{C} 14$ & 3.0 & 0.11 \\
\hline C14:1 & 0.10 & 0.01 \\
\hline $\mathrm{C} 15$ & 1.12 & 0.11 \\
\hline $\mathrm{C} 16$ & 23.38 & 0.95 \\
\hline C16:1 & 4.56 & 0.36 \\
\hline $\mathrm{C} 17$ & 1.01 & 0.14 \\
\hline $\mathrm{C} 18$ & 20.00 & 1.00 \\
\hline C18:1n-12 & 3.59 & 0.58 \\
\hline C18:1n-9 & 30.39 & 1.12 \\
\hline $\mathrm{C} 18: 1 \mathrm{t} 3$ & 0.62 & 0.77 \\
\hline C18:1n-7 & 0.23 & 0.04 \\
\hline C18:1n-5 & 0.35 & 0.02 \\
\hline C18:1 t16 & 0.15 & 0.02 \\
\hline C19 & 0.16 & 0.14 \\
\hline $\mathrm{C} 18: 2 \mathrm{t} 8, \mathrm{c} 12 / \mathrm{c} 9, \mathrm{t} 13$ & 0.54 & 0.11 \\
\hline$C 18: 2 n-6$ & 6.08 & 0.66 \\
\hline C19:1 & 0.01 & 0.01 \\
\hline $\mathrm{C} 20$ & 0.12 & 0.14 \\
\hline C18:3n-6 & 0.00 & 0.00 \\
\hline $\mathrm{C} 18: 3 \mathrm{c} 9, \mathrm{t} 12, \mathrm{c} 15 / \mathrm{t} 9, \mathrm{c} 12, \mathrm{c} 15$ & 0.33 & 0.02 \\
\hline C20:1 & 1.51 & 0.09 \\
\hline C18:3n-3 & 0.00 & 0.00 \\
\hline $\mathrm{C} 18: 2 \mathrm{t} 9, \mathrm{t} 12$ & 0.04 & 0.01 \\
\hline $\mathrm{C} 18: 2 \mathrm{t} 9, \mathrm{c} 12$ & 0.05 & 0.01 \\
\hline $\mathrm{C} 18: 2 \mathrm{c} 9, \mathrm{t} 12$ & 0.08 & 0.01 \\
\hline $\mathrm{C} 18: 2 \mathrm{c} 9, \mathrm{c} 12$ & 0.04 & 0.01 \\
\hline $\mathrm{C} 22$ & 0.04 & 0.01 \\
\hline$C 20: 5 n-3$ & 0.87 & 0.12 \\
\hline$C 22: 4 n-6$ & 0.73 & 0.08 \\
\hline$C 21: 5 n-3$ & 0.01 & 0.01 \\
\hline$C 22: 5 n-3$ & 0.52 & 0.30 \\
\hline$C 22: 6 n-3$ & 0.11 & 0.03 \\
\hline
\end{tabular}

qui de alpaca (5.75 a 5.97, Salvá et al., 2009). Asimismo, la literatura reporta $\mathrm{pH}$ de 5.75 para charqui de cerdos (Choi et al., 2008) y 5.53 a 5.76 para charqui de bovino (Yang et al., 2009).
La medición de la actividad de agua en subproductos alimenticios es importante debido a su estrecha relación con el contenido de agua (Leistner, 1987). En el presente estudio, la actividad de agua del charqui (0.6 a 
Cuadro 3. Proporción de los ácidos grasos (\%) en la grasa intramuscular de charqui de llama

\begin{tabular}{lc}
\hline Ácidos grasos & Proporción (\%) \\
\hline Ácidos grasos saturados (SFA) & 49.49 \\
Ácidos grasos monoinsaturados (MUFA) & 41.57 \\
Ácidos grasos poliinsaturados (PUFA) & 8.94 \\
Ácido Linoleico conjugado (CLA) & 0.21 \\
Omega 3 (n-3) & 1.67 \\
Omega 6 (n-6) & 6.17 \\
Hipocolesterohemicos (h) & 43.08 \\
Hipercolesterohemicos (H) & 26.67 \\
Ácido eicosapentaenoico (EPA) & 0.87 \\
Ácido docosahexaenoico (DHA) & 0.10 \\
n-6/n-3 & 3.70 \\
h/H & 1.62 \\
PUFA/SFA & 0.18 \\
Ácidos grasos deseables (DFA) & 70.27 \\
\hline
\end{tabular}

Cuadro 4. Contenido de minerales en charqui de llama (expresado en $\mathrm{mg} / 100 \mathrm{~g}$ de extracto seco)

\begin{tabular}{lrrc}
\hline Minerales & Promedio & D.E. & Rango \\
\hline Calcio & 71.52 & 11.34 & $58.6-88.0$ \\
Cobre & 2.38 & 0.44 & $1.9-3.1$ \\
Hierro & 11.96 & 3.51 & $8.0-16.9$ \\
Magnesio & 106.24 & 7.39 & $97.4-117.0$ \\
Mangan eso & 0.54 & 0.18 & $0.3-0.8$ \\
Potasio & 1658.80 & 88.00 & $1582-1809$ \\
Sodio & 2434.40 & 576.53 & $1759-2968$ \\
Zinc & 18.88 & 4.88 & $11.1-24.4$ \\
\hline
\end{tabular}

0.7) (Cuadro 5) fue bastante similar al charqui de alpaca $(0.65$, Salvá, 2009) e inferiores al bovino (0.74), cerdo (0.71), pavo (0.77) y búfalo (0.79) (Lonnecker et al., 2010). La actividad de agua es útil para describir el estado de equilibrio termodinámico del charqui
(Labuza, 1980; Rockland y Nishi, 1980), pues debe tener una actividad estable para evitar cambios de calidad durante su almacenamiento (Yamaguchi et al., 1986). El proceso de fabricación tiene un efecto importante sobre la actividad de agua y, por lo tanto, 
Cuadro 5. Características tecnológicas de charqui de llama

\begin{tabular}{lccc}
\hline Característica & Promedio & D.E. & Rango \\
\hline $\mathrm{pH}$ & 5.96 & 0.05 & $5.9-6.1$ \\
Actividad de agua $\left(\mathrm{a}_{\mathrm{w}}\right)$ & 0.64 & 0.05 & $0.6-0.7$ \\
Capacidad de retención de agua (WHC) & & & \\
$\quad$ - Ganancia de peso de rehidratación $(\%)$ & 43.50 & 2.80 & $40.7-47.9$ \\
$\quad$ - Pérdida por cocción $(\%)$ & 22.32 & 3.22 & $18.3-28.8$ \\
$\quad$ - WBSF $\left(\mathrm{kg} / \mathrm{cm}^{3}\right)$ & 5.36 & 0.58 & $4.5-6.1$ \\
Color & & & \\
- $\mathrm{L}^{*}$ & 41.69 & 3.40 & $37.0-46.8$ \\
$\quad$ - $\mathrm{a}^{*}$ & 4.64 & 0.75 & $3.8-6.3$ \\
- $\mathrm{b}^{*}$ & 12.21 & 1.75 & $10.2-15.2$ \\
\hline
\end{tabular}

${ }^{1} L^{*}$ : luminosidad; $a^{*}$ : tenores de rojo-verde; $b^{*}$ : tenores de amarillo-azul

en la calidad del producto durante el almacenamiento. Según Cheftel J y Cheftel H (1992), la inhibición del crecimiento microbiano es total con valores inferiores a 0.65 , por lo que se puede considerar que el charqui tenía una buena estabilidad a temperatura ambiente desde el punto de vista microbiológico.

La capacidad de retención de agua (WHC) en términos de ganancia de peso por rehidratación (43.50\%) y pérdida por cocción $(22.32 \%)$ fue diferente a los valores reportados para charqui de alpaca (50.79 y $13.41 \%$, respectivamente; Salvá, 2009). La WHC es una propiedad importante que determina las pérdidas de peso de la carne, principalmente por liberación de líquidos que se producen en toda la cadena de distribución y transformación de la carne, y supone pérdidas económicas, pudiendo también afectar a la calidad de la carne y de sus subproductos (jugosidad, palatabilidad, etc.). La WHC de carne en fresco de los camélidos es ligeramente menor o similar a la de otras especies (Cristofanelli et al., 2004).

Los valores de fuerza de corte (WBSF) para charqui fueron de $5.36 \mathrm{~kg} / \mathrm{cm}^{3}$, mientras que se reporta valores de $5.48 \mathrm{~kg} / \mathrm{cm}^{3}$ para charqui de cerdo, $5.31 \mathrm{~kg} / \mathrm{cm}^{3}$ el de bo- vino (Yang et al., 2009), $13.70 \mathrm{~kg} / \mathrm{cm}^{3}$ para el de emú y $6.60 \mathrm{~kg} / \mathrm{cm}^{3}$ para el de pavo (Carr et al., 1997). Sin embargo, estudios en carne (Longissimus dorsi) en alpaca cocinado registran valores de $4.67 \pm 0.84 \mathrm{~kg} / \mathrm{cm}^{3}$ (Salvá et al., 2009). La WBSF es un indicador de la terneza, que es definida como la facilidad con que la carne se deja masticar, que puede ser descompuesta en tres sensaciones por el consumidor: una inicial, o facilidad a penetración y corte, otra más prolongada que sería la resistencia que ofrece a la rotura a lo largo de la masticación, y una final, que daría la sensación de residuo (Osório et al., 2009). La fuente de variación de la terneza, se puede atribuir a la edad del animal, sexo, peso, raza y estrés ante mortem (Muchenje et al., 2009). Según Muir et al. (2000) y Monsón et al. (2005), la terneza de la carne es función del contenido de colágeno, la estabilidad térmica y la estructura de las miofibrillas del músculo. De acuerdo a Lepetit (2007), el contenido de colágeno parece estar altamente correlacionado con la fuerza de corte de la carne, aunque esta correlación parece ser baja en la carne cocida.

Los valores de la determinación calorimétrica (Cuadro 5) son ligeramente inferiores a los valores $L^{*}(67.16)$ y $b^{*}$ (14.66) pero 


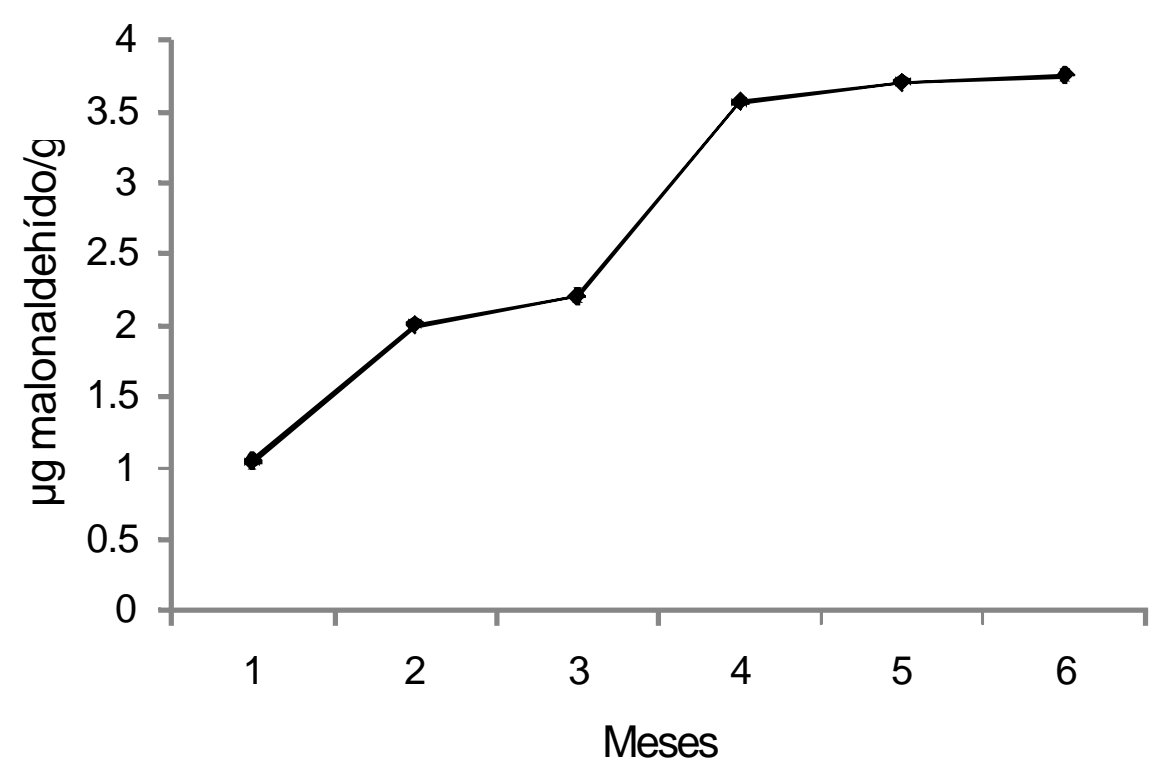

Figura 1. Cambios en los valores de sustancias reactivas al ácido tiobarbitúrico (TBAS, ?g malonaldehido/g de muestra) en charqui de llama almacenado durante seis meses a temperatura ambiente

mayor en $a^{*}$ (3.10) en charqui de alpaca (Salvá, 2009) y diferentes a los de charqui de bovino $\left(\mathrm{L}^{*}=30.66, \mathrm{a}^{*}=13.42, \mathrm{~b}^{*}=4.24\right.$; Konieczny et al., 2007). Se podría decir que el charqui de llama presenta un valor mayor en luminosidad $\left(\mathrm{L}^{*}\right)$ y en el tenor de amarillez $\left(b^{*}\right)$, mientras que el de bovino posee mayor tenor de rojez $\left(\mathrm{a}^{*}\right)$ por el uso de aditivos (nitritos) que permiten mantener el color.

El contenido de TBARS en el primer mes de almacenamiento fue 1.02 ?g malonaldehido/g (Fig. 1), incrementándose gradualmente hasta alcanzar 3.56 en el cuarto mes, y de allí presentando solo un leve incremento. En contraposición, trabajos en charqui de alpaca registran cambios para 12 meses de almacenamiento de 2.93 a 3.77 ?g malonaldehido/g en muestras envasadas al vacío y de 2.93 a 4.28 en muestras envasadas sin vacío (Salvá, 2009). Según Yang et al. (2009), el charqui de cerdo muestra un valor de TBARS (5.25) significativamente más alto y el del bovino el valor más bajo (3.75) entre varios tipos de charqui después de un almacenamiento de 30 días, indicando que el charqui de cerdo es el más susceptible a la oxidación lipídica por el mayor contenido de PUFA.

\section{ConClusiones}

El charqui de llama se caracteriza por:

- Un nivel alto de proteína y baja actividad de agua.

- Grasa con un cociente de ácidos grasos poliinsaturados/saturados y nivel de CLA similar a la carne de alpacas y bovinos criados en sistemas de pastoreo.

- Cocientes de ácidos grasos n-6/n-3 favorables y niveles de ácidos grasos deseables superior al encontrado en otras carnes de rumiantes.

- Nivel de sodio más bajo que el charqui de alpaca pero relativamente más alto que el de bovino.

- Características tecnológicas coincidentes con valores reportados para charqui de alpaca y bovino. 


\section{Literatura Citada}

1. AOAC. 1996. Official methods of analysis. $17^{\text {th }}$ ed. Gaithersburg, MD: Association of Official Analytical Chemists.

2. Bergman I, Loxley R. 1963. Two improved and simplified methods for the spectrophotometric determination of hydroxyoriline. Anal Chem 35: 19611965.

3. $[B D H]$ British Department of Health. 1994. Nutritional aspects of cardiovascular disease. Report on health and social subjects N. ${ }^{\circ}$ 46. London: Her Majesty's Stationery Office.

4. Bohac CE, Rhee KS, Cross HR, Ono K. 1988. Assessment of methodologies for colorimetric cholesterol assay of meats. J Food Sci 53: 1642-1644.

5. Carr MA, Miller MF, Daniel DR, Yarbrough CE, Petrosky JD, Thompson LD. 1997. Evaluation of the physical, chemical and sensory properties of jerky processed from emu, beef, and turkey. J Food Quality 20: 419-425

6. Cheftel JC, Cheftel H. 1992. Introducción a la bioquímica y tecnología de los alimentos. Vol I. Zaragoza: Editorial Acribia.

7. Choi J-H, Jeong J-Y, Han D-J, Choi Y-S, Kim H-Y, Lee M-A, Lee E-S, Paik H-D, Kim C-J. 2008. Effects of pork/ beef levels and various casings on quality properties of semi-dried jerky. Meat Sci 80: 278-286.

8. Coates W, Ayerza R. 2004. Fatty acid composition of llama muscle and internal fat in two Argentinian herds. Small Rum Res 52: 231-238.

9. Costa RG, Malveira AS, Azevedo PS, Ramos do Egypto RC, Madruga MS, Araújo JT. 2009. Lipid profile of lamb meat from different genotypes submitted to diets with different energy levels. $\mathrm{R}$ Bras Zootec 38: 532-538.

10. Cristofanelli S, Antonini A, Torres D, Polidori P, Renieri C. 2004. Meat and carcass quality from Peruvian llama
(Lama glama) and alpaca (Lama pacos). Meat Sci 66: 589-593.

11. Cross HR, Carpenter ZL, Smith GC. 1973. Effect of intramuscular collagen and elastin on bovine muscle tenderness. J Food Sci 38: 998-1003.

12. Duckett SK, Neel JPS, Fontenot JP, Clapham WM. 2009. Effects of winter stocker growth rate and finishing system on: III. Tissue proximate, fatty acid, vitamin, and cholesterol content. J Anim Sci 87: 2961-2970.

13. El-Faer MZ, Rawdah TN, Attar KM, Dawson MV. 1991. Mineral and proximate composition of the meat of the one-humped camel (Camelus dromedarius). Food Chem 42: 139-143.

14. Folch J, Lees M, Stanley S. 1957. A simple method for the isolation and purification of total lipids from animal tissues. J Biol Chem 226: 497-509.

15. Göncü Karakök S, Ozogul Y, Saler M, Ozogul F. 2010. Proximate analysis. Fatty acid profiles and mineral contents of meats: a comparative study. J Muscle Foods 21: 210-223.

16. Hill F. 1966. The solubility of intramuscular collagen in meat animals of various ages. J Food Sci 31: 161-166.

17. Honikel KO. 1998. Reference methods for the assessment of physical characteristics of meat. Meat Sci 49: 447-457.

18. Kadim IT, Mahgoub O, Al-Marzooqi W, Al-Zadjali S, Annamalai $K$, Mansour MH. 2006. Effects of age on composition and quality of muscle Longissimus thoracis of the Omani Arabian camel (Camelus dromedaries). Meat Sci 73: 619-625.

19. Konieczny P, Stangierski J, Kijowski J. 2007. Physical and chemical characteristics and acceptability of home style beef jerky. Meat Sci 76: 253-257.

20. Labuza TP. 1980. The effect of water activity on reaction kinetics of food deterioration. Food Technol 34: 36-42.

21. Leistner L. 1987. Shelf-stable products and intermediate moisture foods based on meat. In: Rockland LB, Beuchat LR 
(eds). Water activity: Theory and applications to foods. New York: Marcel Dekker. p 295-328.

22. Lepetit J. 2007. A theoretical approach of the relationships between collagen content, collagen cross-links and meat tenderness. Meat Sci 76: 147-159.

23. Lonnecker SM, Boyle E, Getty K, Buege D, Ingham S, Searls $G$, Harper N. 2010. Production methods and product characteristics of jerky produced by small and very small meat processing businesses. J Muscle Foods 21: 826-833.

24. Monsón F, Sañudo C, Sierra I. 2005. Influence of breed and ageing time on the sensory meat quality and consumer acceptability in intensively reared beef. Meat Sci 71: 471-479.

25. Muchenje V, Dzama K, Chimonyo M, Strydom P, Hugo A, Raats J. 2009. Some biochemical aspects pertaining to beef eating quality and consumer health: A review. Food Chem 112: 279-289.

26. Muir PD, Wallace GJ, Dobbie PM, Bown MD. 2000. A comparison of animal performance and carcass and meat quality characteristics in Hereford, Hereford x Friesian, and Friesian steers grazed together at pasture. New Zeal J Agric Res 43: 193-205.

27. Osório JC, Moreira MT, Sañudo C. 2009. Características sensoriais da carne ovina. R Bras Zootec 38: 292-300.

28. Quinton RD, Cornforth DP, Hendricks DG, Brennand CP, Su YK. 1997. Acceptability and composition of some acidified meat and vegetable stick products. J Food Sci 62: 1250-1254.

29. Rockland LB, Nishi SK. 1980. Influence of water activity on food product quality and stability. Food Technol 34: 42-51.
30. [RIRDC] Rural Industries Research and Development Corporation. 2007. Composition of new meats, analyses and nutrient composition of innovative meat industries. RIRDC Publication N. ${ }^{\circ}$ 07/ 036. Australia: RIRDC. 59 p.

31. Salvá KS. 2009. Características de la carne y charqui de alpaca (Vicugna pacos). Tesis de Doctorado. España: Facultad de Veterinaria, Universidad de León. 299 p.

32. Salvá KS, Zumalacárregui JM, Figueira AC, Osorio MT, Mateo J. 2009. Nutrient composition and technological quality of meat from alpacas reared in Peru. Meat Sci 82: 450-455.

33. Sinnhuber RO, Yu TC. 1977. The 2thiobarbituric acid reaction, an objective measure of the oxidative determination occurring in fats and oils. J Jpn Soc Fish Sci 26: 259.

34. Tichivangana JZ, Morrissey PA. 1985. Myoglobin and inorganic metals as proxidants in raw and cooked muscles system. Meat Sci 15: 107-116.

35. [USDA] United States Department of Agriculture. 2008. National Nutrient Database for Standard Reference, Release 21. [Internet]. Disponible en: http://www.ars.usda.gov/ba/bhnrc/ndl

36. Yamaguchi $N$, Naito S, Okada $Y$, Nagase A. 1986. Effect of oxygen barrier of packaging material on food preservation. Annual Report of the Food Research Institute, Japan 27: 69-73.

37. Yang H-S, Hwang Y-H, Joo S-T, Park G-B. 2009. The physicochemical and microbiological characteristics of pork jerky in comparison to beef jerky. Meat Sci 82: 289-294. 